\title{
Identificación del impacto de la gestión de los stakeholders en las estructuras de las empresas que desarrollan estrategias de responsabilidad social empresarial Identification of the Impact of Stakeholder Management on Company Structures Developing Corporate Social Responsibility Strategies Identificação do impacto da gestão dos stakeholders nas estruturas das empresas que desenvolvem estratégias de responsabilidade social empresarial
}

\author{
Jorge Roberto Volpentesta* \\ Tomás Chahín \\ Marcelo Fabián Alcaín ${ }^{* * *}$ \\ Gustavo Rodolfo Nievas ${ }^{* * *}$ \\ Héctor Eduardo Spinellit
}

* Doctorando en Administración en la Universidad de Buenos Aires, Facultad de Ciencias Económicas. Magister en Dirección de Empresas de la Universidad de Deusto (El Salvador). Profesor Asociado Interino a cargo de la titularidad de la materia Sistemas Administrativos en la Facultad de Ciencias Económicas de la Universidad de Buenos Aires. Director del grupo de investigación, cuyos integrantes son los demás autores del texto. Correo electrónico: jorge.volpentesta@hotmail.com

** Integrante del equipo de investigación: Profesor adjunto regular. Profesor de la Facultad de Ciencias Económicas, Universidad de Buenos Aires. Correo electrónico: tomaschahin@gmail.com

*** Integrante del equipo de investigación: Profesor adjunto regular. Profesor de la Facultad de Ciencias Económicas, Universidad de Buenos Aires. Correo electrónico: marceloalcain@gmail.com

***** Integrante del equipo de investigación: Profesor adjunto interino. Profesor de la Facultad de Ciencias Económicas, Universidad de Buenos Aires. Correo electrónico: grnievas@hotmail.com

****** Integrante del equipo de investigación: Profesor adjunto interino. Profesor de la Facultad de Ciencias Económicas, Universidad de Buenos Aires. Correo electrónico: hectorspinelli@fibertel.com 


\section{María Inés Cordero \\ Alicia Cortejarena $a^{* *+* * * *}$ \\ Penélope Greco ${ }^{\star \star \star \star \star \star \star \star \star ~}$}

Fecha de recibido: 8 de julio de 2013

Fecha de aprobado: 21 de diciembre de 2013

Doi: dx.doi.org/10.12804/rev.univ.empresa.26.2014.02

Para citar este artículo: Volpentesta, J. R., Chahín, T., Alcaín, M. F., Nievas, G. R., Spinelli, H. E., Cordero, M. I., Cortejarena, A., Greco, P. (2014). Identificación del impacto de la gestión de los stakeholders en las estructuras de las empresas que desarrollan estrategias de responsabilidad social empresarial. Universidad \& Empresa, 16(26), 65-94.doi: dx.doi.org/10.12804/rev.univ.empresa.26.2014.02

\section{RESUMEN}

Sobre una muestra de veinticinco empresas que actúan en territorio argentino y que manifiestan tener con la sociedad un compromiso que va más allá de la obtención de utilidades para sus accionistas, esta investigación ha procurado 1) indagar acerca de los mecanismos administrativos (comunicacionales, procedimentales, organizativos y estructurales) que ellas adoptan para poder diferenciar, escuchar y traducir las voces de sus stakeholders en acciones concretas, con el fin de satisfacer los requerimientos de los mismos, 2) inquirir si esos mecanismos responden a propuestas provenientes de organizaciones que tienen formulaciones de modelos al respecto o si son desarrollos originales surgidos de la cultura y las características de gestión de la propia empresa y 3) evaluar la efectividad de dichos mecanismos a través de la incorporación concreta de los requerimientos de los stakeholders en la visión, misión, objetivos, políticas y estrategias de las empresas, analizando la congruencia o discrepancia de lo que la empresa manifiesta y lo que ella hace realmente.

Palabras clave: Administración, grupos de interés, responsabilidad social empresarial.

\section{ABSTRACT}

In a sample of twenty-five companies operating in Argentinian territory that express having a commitment with society that goes beyond obtaining profit for their shareholders, this research has endeavored 1) to find out about the management mechanisms (communicational, procedural, organizational and structural) they adopt to be able to differentiate, listen to and translate the voices of their stakeholders into concrete actions in order to meet their

\footnotetext{
******* Integrante del equipo de investigación: Jefe de trabajos prácticos. Profesor de la Facultad de Ciencias Económicas, Universidad de Buenos Aires. Correo electrónico: maria.cordero@hsbc. com.ar

******** Integrante del equipo de investigación: Ayudante de $1^{\circ}$. Profesora de la Facultad de Ciencias Económicas, Universidad de Buenos Aires. Correo electrónico: acortagerena@gmail.com

${ }^{* * * * * * * * *}$ Integrante del equipo de investigación: Ayudante de $1^{\circ}$. Profesora de la Facultad de Ciencias Económicas, Universidad de Buenos Aires. Correo electrónico: penelopegreco@gmail.com
} 
requirements; 2) to inquire whether these mechanisms respond to proposals from organizations that have formulations of models in this regard, or if they are original developments that are the result of the culture and management characteristics of the company itself; and 3) to evaluate the effectiveness of these mechanisms by means of concrete incorporation of the requirements of the stakeholders in the vision, mission, objectives, policies and strategies of the companies, by analyzing the consistency or discrepancy of what the company states and what it does in reality.

Keywords: Corporate social responsibility, Management, Stakeholders.

\section{RESUMO}

Sobre uma amostra de vinte e cinco empresas que atuam em território argentino e que manifestam ter com a sociedade um compromisso que vai além da obtenção de utilidades para seus acionistas, esta pesquisa tem procurado: 1) indagar acerca dos mecanismos administrativos (comunicacionais, procedimentais, organizativos e estruturais) que elas adoptam para poder diferenciar, ouvir e traduzir as vozes de seus stakeholders em ações concretas, com o fim de satisfazer os requerimentos dos mesmos; 2) inquirir se esses mecanismos respondem a propostas provenientes de organizações que têm formulações de modelos ao respeito ou se são desenvolvimentos originais surgidos da cultura e as características de gestão da própria empresa, e 3) avaliar a efetividade de ditos mecanismos através da incorporação concreta dos requerimentos dos stakeholders na visão, missão, objetivos políticas e estratégias das empresas, analisando a congruência ou discrepância do que a empresa manifesta e o que ela faz realmente.

Palavras-chave: administração, grupos de interesse, responsabilidade social empresarial.

\section{INTRODUCCIÓN}

El término stakeholder es un neologismo anglosajón que normalmente se traduce al español como: interesado, grupo de interés o de interesados, o como parte interesada (International Organization for Standardization, ISO 26000, 2010). Los stakeholders son definidos como todas aquellas personas, grupos, empresas, comunidad y sociedad que tienen interés en la existencia y desarrollo de una empresa. Son interesados directos e indirectos con poder real o potencial para influir en las decisiones empresariales como, por ejemplo, empleados, clientes, proveedores, accionistas/inversionistas, organismos públicos, asociaciones profesionales, grupos religiosos, comunidad, organizaciones de la sociedad civil, ong y el público en general.

La idea central que guio esta investigación surge de la evidencia de que un número creciente de empresas realiza actividades que favorecen la incorporación dentro de sus visiones, misiones, objetivos y 
estrategias, de consideraciones tendientes a la satisfacción de expectativas, deseos, intereses y demandas que van más allá de la exclusiva maximización de la riqueza de sus accionistas o propietarios, es decir, que consideran los objetivos de sus stakeholders.

La evolución de este proceso genera argumentaciones que impactan sobre los procesos de gestión y, de manera directa e indirecta, también sobre el gobierno de las empresas. El fenómeno de incorporación en ellas de objetivos de interesados no accionistas es producto de una evolución de la forma de "ser" empresa que algunas de estas compañías han adoptado y que permite reconocer que hoy, arquetípicamente y simplificando el análisis, esas organizaciones pueden dividirse entre aquellas que siguen un modelo de gobierno accionarial o financiero puro $\mathrm{y}$ aquellas que siguen un modelo pluralista o gobierno de stakeholders.

La incorporación de objetivos de grupos de interesados no accionariales refiere a un proceso que ha llevado a que algunas empresas reconozcan a diversos stakeholders no propietarios y a sus intereses y, de manera no uniforme, les permitan participar de ciertos procesos de toma de decisiones. Indagar sobre esos procesos y las características de incorporación de los stakeholders a la toma de decisiones son los objetivos básicos de este trabajo.

\section{OBJETIVOS}

En el desarrollo de la gestión empresarial socialmente responsable, una de las tareas más complejas es la creación de relaciones de confianza con los stakeholders. Para esto resulta necesario que cada empresa pueda diferenciar sus grupos de interesados de manera que, una vez escuchadas sus demandas y expectativas y definidos sus intereses y pedidos, estos puedan ser incorporados en su visión, misión, objetivos, estrategias y políticas.

Estar de acuerdo con lo anterior implica aceptar un cambio en la forma de gerenciamiento de las empresas, en especial, en relación con los intereses que son tenidos en cuenta cuando se dirige una compañía. Esto es así puesto que en los sistemas de gestión organizacional contemporáneos prevalecen, generalmente, más unos intereses que otros. Dicho de otra forma, se privilegian los intereses que detentan algunos stakeholders frente a los de otros. Por ejemplo, una empresa con un fuerte enfoque en el mercado privilegiará los intereses de sus accionistas y de sus clientes. En tanto que una organización de la sociedad civil ponde- 
rará usualmente más los intereses de sus empleados y de la comunidad.

Dirigir una empresa dentro de los lineamientos de la responsabilidad social empresarial y en sintonía con el nuevo paradigma del desarrollo sostenible implica incorporar en el análisis estratégico el debate sobre qué intereses son los que deberían prevalecer, o bien, no ser marginados, si es que se piensa en la sustentabilidad de la empresa en el largo plazo. Esto dado que entre los doctrinarios $\mathrm{y}$ en la sociedad misma aumenta cada vez más el consenso respecto a que la desestimación y marginación sistemáticas de algunos intereses, en beneficio de otros, compromete seriamente la supervivencia de la empresa en el largo plazo.

Esta nueva conceptualización del gerenciamiento de las organizaciones empresariales permite ampliar los campos de racionalidad que intervienen en el diseño de su estructura de poder. De esta manera es posible encontrar empresas que se preocupen por considerar a sus stakeholders más relevantes, con los cuales asumen compromisos en el largo plazo, atendiendo al esquema de poder que en cada situación se presenta. Este enfoque se contrapone a las visiones reduccionistas que concentran la actuación empresarial, exclusivamente, en los inte- reses de los accionistas, en un caso, o en los intereses de los clientes, en algún otro. Además, en las organizaciones se juegan múltiples intereses competitivos, por lo general conflictivos, en una circunstancia espaciotemporal en la que los stakeholders actúan en diferentes posiciones de poder. Ellos intervienen en los arreglos organizacionales o pactos que realizan las diferentes coaliciones que detentan el poder - en especial, entre los grupos de interés primarios, que son los que sostienen principalmente la organización-, los cuales son determinantes para la supervivencia de la organización.

Una vez identificados los stakeholders relevantes para una organización hay que desarrollar canales de comunicación que permitan que sus voces sean escuchadas, en especial las de aquellos que temporalmente no tienen mucho poder. Cada grupo de interesados, por otro lado, se presenta con una particular forma de efectuar sus demandas, reclamaciones y objetivos, los que serán comunicados a las empresas de manera idiosincrásica, esto es, con su particular estilo y modalidad. A las dificultades que plantea esta situación, se suma el hecho de que las vinculaciones entre la empresa y los distintos grupos de stakeholders deben ser dimensionadas dentro de la complejidad que ellas presentan. 
Una de ellas, es la presencia de permanentes cambios dentro de los grupos y de sus intereses y expectativas; una circunstancia que en ocasiones genera ambigüedad en la comunicación. Además, las posiciones de los diferentes grupos $-\mathrm{y}$ aun las de las empresas- pueden ser antagónicas o encontrar muy pocos puntos de coincidencia.

Finalmente, la empresa se ve enfrentada a incorporar esas voces dentro de los mecanismos de formación de su misión, visión, objetivos, políticas y estrategias. De esta manera, solo volcando en las prácticas cotidianas los requerimientos de los stakeholders, podrá configurarse un gobierno empresarial que considere realmente sus intereses.

La realidad indica que la cantidad de stakeholders de una empresa, al considerarlos a la manera clásica, es muy grande, pues ella tiene como límite utópico a todos los que se interesan o interactúan con la misma. El problema reside en cómo materializar en la práctica un modelo de gobierno de los stakeholders cuando es tan amplia la necesidad de información, son tan variados los enfoques y tan numerosos y divergentes los intereses que se presentan.

En consecuencia, dentro del planteamiento de este nuevo modelo de gobierno empresarial la pregunta que surge es ¿cuáles son los mecanismos administrativos que utilizan las empresas para cristalizar operacionalmente el modelo de gobierno de los stakeholders con el fin de tratar de considerar a cada uno de los requerimientos que ellos tienen? Es esperable que la empresa considere las expectativas e intereses de aquellos más relevantes, pero no por ello podrá postergar o desestimar las demandas y peticiones de aquellos otros grupos de interesados que, circunstancial y temporalmente, son menos relevantes.

Existen numerosas aportaciones doctrinarias - principalmente provenientes de países europeos y, en particular, de los Estados Unidos - respecto a cuáles son los mecanismos a través de los que la dirección de la empresa se vincula con sus stakeholders, escucha sus opiniones y vuelca en sus instrumentos administrativos hacia sus deseos y necesidades. Sin embargo, dentro de la literatura, en un país como Argentina, no se cuenta con investigaciones que permitan determinar cuáles son los mecanismos que realmente utilizan las empresas ubicadas en su territorio, ni cuáles son sus características.

Por este motivo, el primer objetivo que guio esta investigación fue: 
Indagar, dentro de aquellas empresas que actúan en territorio argentino y que dicen tener con la sociedad un compromiso que va más allá de la obtención de utilidades para sus accionistas, las construcciones administrativas - comunicacionales, procedimentales, organizativas, estructurales, entre otras-que ellas adoptan para diferenciar, escuchar y traducir las voces de sus stakeholders en acciones concretas que permitan satisfacer sus requerimientos.

Una vez hecha una indagación inicial acerca de estos mecanismos, se propuso como segundo objetivo de la investigación lo siguiente:

Especificar si los mecanismos utilizados responden a propuestas provenientes de organizaciones que tienen formulaciones de modelos al respecto, por ejemplo, las planteadas por el Institute of Social and Ethical Accountability (AA1000S) o las del Global ReportingInitiative (GRI), o si son desarrollos originales surgidos de la cultura y las características de gestión de la propia empresa.

Finalmente, como tercer objetivo se planteó lo siguiente:

Evaluar la efectividad de esos mecanismos a través de la incorporación de los requerimientos de los stakeholders en la visión, misión, objetivos, políticas y estrategias de las empresas, analizando la congruencia o discrepancia de lo que la empresa dice frente a lo que ella hace en realidad; confrontando para esto, por ejemplo, los enunciados de su visión, misión y objetivos con las acciones y decisiones que efectivamente la compañía asume en su diario vivir.

La proposición de investigación, asociada a estos objetivos, es la siguiente:

Si las empresas operan en su relación con los stakeholders, a través de mecanismos administrativos - ya sean tomados de organizaciones especializadas o de desarrollo propio-que permitan su identificación, comunicación y el posterior volcado de sus intereses en su operación, no debería existir discrecionalidad por parte de los directivos de esas compañías para la determinación de qué intereses son los que se debería satisfacer.

\section{Revisión de la literatura}

En función del nuevo paradigma propuesto por el concepto del desarrollo sostenible - progreso y desarrollo social, equilibrio ambiental y crecimiento económico (Elkington, 1994) - y del afianzamiento en las empresas de actuaciones enmarcadas dentro de la Responsabilidad Social Empresarial (RSE), las compañías que desean ser efectivas en los exigentes y competitivos mercados actuales y futuros no podrían ignorar a sus stakeholders, 
tanto internos como externos - primarios como secundarios-. Ellas deberían considerar ajustar sus estrategias, políticas, objetivos y sus enunciados de visión y misión, para reflejar las preocupaciones de todos ellos.

En los últimos años, y en virtud del impulso generado por conceptos como los de desarrollo sostenible y actuaciones de RSE, la vinculación de las empresas con cada uno de sus stakeholders dejó de ser una actividad de escasa importancia para alcanzar el éxito competitivo. En la actualidad, esta es conceptualizada como un elemento diferencial en el posicionamiento estratégico y en la posibilidad de supervivencia de las empresas. De hecho, cada grupo de interesados cuenta con una determinada capacidad potencial -en mayor o menor medida para cada grupo y, a su vez, cambiante a través del tiempo- para amenazar de algún modo a las empresas o para cooperar con ellas.

Ante esta realidad, cada empresa trata de operar para disminuir la posibilidad de amenazas - o directamente, la realización de acciones hostiles - mientras intenta también aumentar los comportamientos colaborativos con sus más importantes stakeholders (Sturzenegger, Vidal Flores, \& Sturzenegger, 2003). Es por ello que resulta primordial para cada empresa diferenciar debidamente a todos sus stakeholders y determinar, de algún modo, el potencial de cooperación o amenaza que ellos detentan (Mitchell, Agle, $\&$ Wood, 1997).

Todas estas consideraciones sobre los stakeholders son tenidas en cuenta por las empresas cuyos propietarios o gestores les asignan un papel importante dentro de su proceso de desarrollo sostenible. Esto se manifiesta en una gestión que atiende los intereses de los stakeholders, de aquellos que influyen o son influenciados por la organización. Esta manera de entender la forma de ser empresa supone un cambio sustancial en la concepción de su gobierno y, en especial, en los valores que guían su acción y los objetivos que persigue.

En función de la consideración que una empresa hace de los intereses de sus distintos stakeholders, es posible identificar dos modelos diferentes de gobierno. Uno de ellos se denomina "modelo de gobierno de los accionistas". Este modelo está representado por el concepto tradicional de gobierno empresarial y se caracteriza por perseguir la maximización del valor de la empresa, satisfaciendo con esto a los inversores. Esta maximización supone 
la concreción del valor central que guía esta filosofía: el crecimiento económico. El modelo centra su atención en favorecer y privilegiar a los accionistas y sus intereses a través del aumento del valor de la empresa, lo que a su vez se ve reflejado en los mercados financieros.

Por su parte, el otro modelo, denominado "gobierno de los stakeholders", persigue un objetivo más amplio que el de satisfacer únicamente los intereses del grupo de los accionistas, ya que su orientación es hacia la obtención de un equilibrio en la satisfacción de los intereses de todos los stakeholders de la empresa, entre los cuales se incluyen, por supuesto, aquellos de los accionistas. Desde la perspectiva de este modelo, el valor central no es el crecimiento económico sino el desarrollo sostenible. El gobierno de la empresa sostenible busca crear valor de forma duradera, tanto para los accionistas como para la sociedad en su conjunto, a través de la satisfacción no solo de los objetivos de los inversionistas, sino de todas las partes interesadas. La esencia de este modelo se fundamenta en la creencia de que no es posible crear valor si las acciones se dirigen exclusivamente a la satisfacción de los intereses de los inversionistas, ignorando o desestimando a todos los demás interesados necesarios para que la organización desarrolle su actividad (Ricart, Rodríguez, Blasco, Elorriaga, \& Castilla, 2002).

Tanto los economistas clásicos como los neoclásicos presentan un modelo de empresa en el que prevalece, en términos generales, una función de producción conforme a una tecnología dada. En este predomina el concepto de la eficiencia técnica, que está orientada por parámetros de costos y decisión tendientes a la maximización del beneficio. Dentro de dicho marco de ideas no sorprende que muchos doctrinarios (Argenti, 1970; Hayek, 1960/1985; Jensen, 2002) expresen que el objetivo básico de la empresa es maximizar el rendimiento a largo plazo del capital que ha sido confiado a los directivos por parte de los accionistas. Estas ideas respecto al objetivo de las empresas se enmarcan dentro de las llamadas teorías monistas, las cuales, en su planteamiento más tradicional, desconocen cualquier otro objetivo distinto a la exclusiva obtención de beneficios para los propietarios.

En los últimos años, sin embargo, otras voces se han hecho escuchar al respecto. Stiglitz (2004), por ejemplo, enfatiza que maximizar la riqueza de los accionistas no necesariamente conduce a la eficiencia económica ni promueve el logro de 
otros objetivos significativos como la protección del medio ambiente, la justicia social y el respeto por los derechos humanos. Este economista aboga por una actuación socialmente responsable de las empresas para compensar algunos fallos del mercado, tales como la información imperfecta, los mercados incompletos y la limitada capacidad de regulación de los Estados para mitigar las externalidades empresariales negativas.

De acuerdo con lo expresado por Rodríguez (2002, p. 76), estas voces plantean una visión pluralista en la que el gobierno empresarial se observa desde una perspectiva más amplia: como una red de estructuras, reglas y procedimientos institucionales que determinan el poder de control, la generación de incentivos a diversos stakeholders-y no solo a los accionistas-, la distribución del riesgo, la orientación a la creación de riqueza para todos los partícipes y la distribución del excedente obtenido por la empresa. Una concepción muy cercana a la tradición empresarial centroeuropea, escandinava e, incluso, japonesa. Esta visión del gobierno de las empresas se plantea dentro de la teoría de los stakeholders, que entiende a la empresa como una unidad que, a través de la producción de bienes y servicios, busca satisfa- cer los deseos, intereses y expectativas de los diferentes grupos que la componen y de los que de alguna forma depende para su supervivencia. Si bien cada stakeholder tiene sus propios objetivos, estos solo podrán lograrse por medio de su compromiso con la acción conjunta ejercida a través de la empresa.

Esta forma de ver el gobierno de las empresas no representa una visión idílica. Por el contrario, ella implica un gran esfuerzo destinado a compatibilizar incentivos y posturas divergentes, arbitrar entre intereses, generar adhesiones y convencer a los otros. Todo esto se hace utilizando criterios de razonabilidad y satisfacción general para lograr algo más que el establecimiento de reglas unilateralmente definidas en función de un único interés. Pfeffer y Salancik (1978/2003, p. 42-43) señalan que este tipo de esfuerzo procura, en lo fundamental, construir redes de intereses y practicar estrategias de cooperación, reforzando la supervisión mutua de todos los diferentes grupos de stakeholders. Ello permite aportar a la solución de los problemas de interdependencia e incertidumbre, al compatibilizar la eficiencia económica con la equidad, por medio de un entramado del que no solo hacen parte los derechos, sino también las responsabilidades individuales y colectivas. 
Frente a la perspectiva de un gobierno pluralista se han generado diversos cuestionamientos. Los dos principales argumentos expresados se centran, por un lado, en la dificultad para definir claramente quiénes son los stakeholders de una empresa $y$, por el otro, para determinar los mecanismos que se utilizan con el fin de combinar y equilibrar sus diferentes deseos e intereses. Sobre la primera cuestión, es necesario saber que los stakeholders de una empresa deben ser definidos por cada compañía en particular, ya que si esta actividad se hace en lo abstracto, adoptando apresuradamente una definición muy amplia de ellos, su cantidad sería ilimitada. Respecto al segundo aspecto, vinculado con las estructuras y los procedimientos institucionales que las empresas diseñan para un efectivo gobierno pluralista, es necesario reconocer que, en función de los diferentes entornos dinámicos y cambiantes que enfrenta cada empresa, resultaría utópico determinar un único modelo de participación de los stakeholders en sus procesos de toma de decisiones.

Cualquiera sea el camino elegido, lo anterior no implica que el protagonismo que los stakeholders puedan lograr en las instancias de gobierno de la empresa conduzca a eliminar el papel y la intervención de los accionistas. Para autores como Rodríguez (2002) vale la pena pensar en situaciones muy diversas que se encuentran en el origen de las múltiples soluciones que pueden llegar a ser aplicables en la práctica organizacional. Entre ellas, se encuentran las siguientes:
Asignación de derechos de propie- dad formales; presencia con voto en el consejo de administración; posibi- lidad de veto a través de la participa- ción en algún comité de supervisión y control; capacidad para designar, proponer, recomendar u oponerse al nombramiento de un cierto número de miembros del consejo de admi- nistración o de un órgano análogo; procedimientos de información y consulta; derecho a participar en el excedente empresarial; percepción de compensaciones en determinadas circunstancias; etc. (p. 93).

Gestionar una empresa en función del modelo de gobierno de los stakeholders implica que los integrantes de sus órganos de dirección deben contemplar, entre sus tareas, las siguientes: priorizar el diálogo con todos los stakeholders, establecer relaciones cordiales entre estos y la empresa, cuidar que los valores asumidos por la empresa sean internalizados por todos sus integrantes, comunicar tanto hacia dentro como hacia afuera esos valores, a través de acciones concretas y evitando el uso 
de lemas o frases huecas, generar mecanismos que permitan identificar adecuadamente a sus stakeholders, así como herramientas que logren incorporar sus intereses a los objetivos empresariales. Ellos deben monitorear, además, que el aprendizaje, el conocimiento y la credibilidad que se generen como resultado de esas tareas aporten al logro de innovaciones, que sean económica, medioambiental y socialmente valiosas (Ricart et al., 2002).

Así, para que se instale en una empresa un gobierno de los stakeholders, resulta necesario generar determinados mecanismos administrativos - comunicacionales, procedimentales, organizativos, estructurales, entre otros - que permitan 1) identificar claramente a los grupos de interés, 2) revelar sus deseos y requerimientos y 3) incorporar esos deseos y requerimientos dentro de la operación administrativa y estratégica de la empresa (Volpentesta, 2011). El estado del arte sobre este campo no permite dilucidar entonces respuestas adecuadas frente a estas últimas cuestiones. Por ello se considera necesario indagar aún más sobre estos aspectos en el mundo de las empresas. Esto con el fin de detectar las modalidades adoptadas en ellas, en esa materia, y debatir también, desde este contexto, la efectividad de las mismas.

\section{METODOLOGÍA}

Este trabajo pertenece al campo de las investigaciones descriptivas. A través de él se busca saber cómo es y cómo se manifiesta el fenómeno estudiado, para el caso de este estudio: el alcance, profundidad y características de la participación de diversos stakeholders en los procesos de gobierno de las empresas. Según Fassio, Pascual y Suárez (2004, p. 40) el objetivo de un diseño descriptivo es describir situaciones o fenómenos, buscando medir los ejes temáticos relevantes de manera independiente. En este tipo de diseño hay que identificar tanto los ejes temáticos principales, o más significativos, como los secundarios. Se trata de interpelar la realidad a través de preguntas del siguiente tipo: “¿cómo es este fenómeno?, ¿cómo se manifiesta?, ¿qué características tiene?, ¿dónde se manifiesta?”.

En el estudio se trabajó con empresas ubicadas en el territorio argentino que manifiestan considerar en sus acciones los requerimientos e intereses no solo de sus propietarios sino también los de otros grupos de interesados. Para la elección de las empresas, como unidades de análisis, se utilizó la técnica de muestreo no probabilístico, entre aquellas que declararon tener actuaciones socialmente responsables a través de in- 
formes o reportes públicos. Sobre la base de la experiencia adquirida en otras investigaciones, en las que también las unidades de análisis fueron empresas, se trabajó con el criterio de muestras disponibles o accesibles en aquellas que aceptaron la invitación de participar en el estudio. Se hizo la solicitud a setenta y ocho empresas, de las cuales veinticinco aceptaron participar en la investigación. Esto representa un porcentaje del $32,05 \%$.

Para la recolección de los datos se realizaron entrevistas semiestructuradas con los responsables de las áreas o sectores encargados de desarrollar las actividades de RSE o de dirigir y/o coordinar dichas acciones. Además, se analizó documentación pública de las empresas consultadas con el fin de verificar el grado de congruencia o discrepancia entre lo que la empresa manifiesta y lo que realmente hace. Para ello se confrontaron, por ejemplo, los enunciados de su visión, misión, objetivos y estrategias con las acciones y decisiones que efectivamente realizaron en el cotidiano, implementando un método de cotejo de la información desde diferentes puntos de referencia (triangulación metodológica).

El trabajo de análisis sobre los datos se hizo inmediatamente después de recolectados. Se organizaron las ideas que de ellos iban emergiendo a través del análisis cualitativo. Esto se hizo con el propósito de establecer conceptos relacionados con el objeto de estudio y de generar nueva teoría, para tratar de aportar al avance del conocimiento en este campo (Taylor \& Bogdan, 1987; Valles, 2000).

\section{RESULTADOS}

Los resultados obtenidos fueron agrupados a partir de las categorías que trascendieron en el análisis de los datos. De esta manera, se agrupan dentro de los siguientes conceptos: diferenciación de los stakeholders, mecanismos de identificación y escucha, participación de los stakeholders en los mecanismos organizacionales, el pensar, el decir y el hacer, consideración estratégica de la actuación socialmente responsable y voluntariedad de las acciones.

La mayor parte de las categorías establecidas se vinculan con los objetivos manifestados para este trabajo. Sin embargo, también se incorporaron aquellas que surgieron de los propios datos y resultaron ser relevantes para los fines del conocimientode las temáticas tratadas. Una circunstancia habitual en estudios con características cualitativas. 


\section{A. Diferenciación de los stakeholders}

Como cada empresa actúa en un determinado contexto, es su responsabilidad establecer los mecanismos para diferenciar a sus stakeholders y sus respectivos intereses. Esta no siempre es una tarea sencilla, debido a 1) la complejidad de las relaciones de las empresas con sus stakeholders, 2) un stakeholder particular puede desempeñar diferentes roles para una misma empresa $y$ 3) diferentes grupos o stakeholders individuales pueden establecer interconexiones en forma de coaliciones que les permitan potenciar algunas de sus características.

Todas las empresas que participaron en la investigación tienen establecidos quiénes son sus stakeholders. El primer aspecto destacable que surge de este hecho consiste en que todas estas empresas los designan nominalmente: clientes, proveedores, comunidad, gobierno, medios de comunicación, organizaciones no gubernamentales, entre otros. Si bien implícitamente el orden en el que ellos se identifican y ordenan en cada empresa sugiere un criterio de importancia, lo cierto es que ninguna compañía tiene una diferenciación cualitativa de ellos o una clasificación de acuerdo a otros criterios.
La literatura sobre este campo es prolífica en clasificaciones de los stakeholders. Entre otras, se encuentran categorías como primarios, secundarios, normativos, derivados, demandantes e influyentes. Estas categorías son indicativas de la importancia y la legitimidad que cada stakeholder tiene para la empresa. Considerando lo anterior, se puede concluir que para estas empresas, al menos en los hechos verificados, todos sus stakeholders tienen un mismo peso relativo, en otras palabras, una misma importancia y relevancia.

En ninguna empresa se clasifica a los stakeholders por niveles o grupos, tal como se encuentra en la literatura, por ejemplo, en los trabajos de Ricart et al. (2002), quienes identifican la existencia de tres niveles, a saber: consustancial, contractual y contextual.

Otro aspecto que se destaca radica en que en ninguna empresa se definieron tampoco atributos que dieran cuenta de una clasificación más refinada como, por ejemplo, la ofrecida por Mitchell et al. (1997). Estos autores elaboraron un modelo dinámico de diferenciación en el que los stakeholders afectan o son afectados por los objetivos o resultados de una organización determinada, en diferente grado, y en la 
medida en que posean entre uno y tres atributos básicos, a saber:

1. El poder del stakeholder para influenciar a la empresa.

2. La legitimidad de la relación del stakeholder con esta.

3. La urgencia de los reclamos del stakeholder hacia la compañía.

La importancia de tener mayor refinamiento en la diferenciación de los stakeholders es que esto facilita la determinación fehaciente de quién, o qué, cuenta realmente para la organización. Además, en todas las empresas los stakeholders fueron definidos en un momento determinado en el tiempo y nunca, posteriormente, esa definición fue modificada o alterada. Esto reafirma la ausencia de elementos cualitativos en su diferenciación, a pesar de que los factores que definen a los stakeholders son dinámicos y se alteran con el paso del tiempo y que entre estos actores pueden también generarse coaliciones e interrelaciones que modifiquen el contexto organizacional.

Otro elemento diferencial que surge del análisis de los datos recolectados es la variación en el número de stakeholders que cada empresa posee. Algunos de ellos aparecen identificados invariablemente en todas las empresas: clientes, provee- dores y empleados. Esta circunstancia no resulta casual, puesto que al estar el desarrollo de las ideas de RSE y de gestión de stakeholders en las empresas argentinas en un estado embrionario, la orientación hacia estas actividades se racionaliza y vincula de manera cercana al negocio principal. Así, tanto los clientes como empleados y proveedores responden a la primera definición de stakeholders dada por el Stanford Research Institute, como: "aquellos grupos sin cuyo apoyo la organización podría dejar de existir" (Freeman 1984, p. 31). Esto se vincula con la necesidad de las empresas de conocer tanto su contexto como los elementos componentes de sus variables controlables. Una evidencia de este pensamiento es lo dicho por el responsable de la Oficina de Asuntos Sociales de la empresa $\mathrm{n}^{\circ} 24$, quien indica:

Buscamos cuáles son los grupos de personas o individuos que se ven alcanzados por las decisiones que toma la compañía y que, con su toma de decisiones, impactan en nuestro negocio (Entrevista realizada el 28 de septiembre de 2012).

Como dato saliente del análisis se tiene que en una sola de las empresas se identifica un stakeholder no humano: el medioambiente. Si bien entre los doctrinarios existen posturas encontradas en la defini- 
ción del medioambiente como stakeholder (Fassin, 2012), lo cierto es que en la República Argentina la legislación positiva relativa al entorno natural se encuentra en un grado muy avanzado, de manera que resulta probable que las empresas no identifiquen este aspecto como uno de especial atención por fuera de las leyes y reglamentos. Lo llamativo de la empresa que identificó el medioambiente como stakeholder, la ${ }^{\circ} 07$, es que su actividad consiste en prestar servicios y, en el desarrollo de su actividad, no hay impacto ambiental significativo.

Las empresas que mayor número de stakeholders identificaron fueron cuatro. El listado de ellos, para cada empresa, es el siguiente:

- Empresa ${ }^{\circ}$ 06: Empleados, asesores externos, productores, asociados (clientes), comunidad, proveedores, Gobierno, ONG.

- Empresa n 07: Clientes, asociados, candidatos, gobierno, comunidad, empleados, medioambiente, proveedores.

- Empresa $n^{\circ}$ 13: Público interno (empleados), proveedores, clientes, consumidores, comunidad, medioambiente, gobierno, sociedad.

- Empresa n ${ }^{\circ}$ 14: Productores, acopiadores, exportadores, industria semillera, gobierno, instituciones relacionadas con el campo, medios de comunicación, empleados y potenciales empleados, inversores, comunidades rurales, proveedores e instituciones educativas.

Otro hecho destacable radica enque solo dos empresas identificaron como stakeholders a los accionistas, en una sola, a las generaciones futuras y, en ninguna, a los gerentes y directivos o a los competidores. Si bien todas las empresas manifiestan tener como stakeholders a sus empleados, ninguna de ellas nombró a sus representantes sindicales, a pesar de que, por el tamaño de las empresas estudiadas, sus trabajadores tienen, de acuerdo con la ley argentina, una representación sindical interna. En la profundización de las preguntas se verificó esta circunstancia. Sin embargo, la mención como stakeholder siempre fue hacia los "empleados" y no hacia los "representantes sindicales".

\section{B. Mecanismos administrativos de identificación y escucha}

La mayoría de las empresas estudiadas declara utilizar diferentes mecanismos de diferenciación de sus stakeholders, con una característica coincidente respecto de su implementación original: en la mayoría de los casos se instalaron como reac- 
ción a demandas específicas o a reclamos concretos de un stakeholder.

La totalidad de las empresas manifestó que el mecanismo utilizado para diferenciar y, principalmente, escuchar a sus stakeholders es el diálogo implementado a través de grupos de interesados. Todas las empresas sustentan esta elección sobre la base de recomendaciones establecidas en normas emanadas por instituciones internacionales como las del Institute of Social and Ethical Accountability (AA1000S) y las del Global Reporting Initiative (GRI) a las que, en su gran mayoría, adhieren explícitamente. Este mecanismo de diálogo es utilizado en especial con el stakeholder "comunidad" o "sociedad", si bien se extiende su uso hacia otros grupos de stakeholders: gobierno, ONG, empleados.

Algunos mecanismos utilizados parecieran estar especializados en determinado tipo de stakeholders, tal es el caso de las encuestas, que prevalecen como procedimiento en la escucha de los empleados y el público interno, o de los desayunos de trabajo, que invariablemente son utilizados con los proveedores.

Una gran mayoría de las empresas estudiadas ha puesto a disposición de algunos stakeholders sitios en
Internet para que ellos, especialmente los que se encuentran fuera de la empresa, puedan manifestar allí sus opiniones y comentarios. Este canal, sin embargo, todavía no es muy utilizado.

Otros mecanismos que las empresas tienen implementados, pero en menor medida, son las reuniones con representantes, los focus groups, las reuniones públicas, la investigación de mercados, el análisis del mapa de públicos y los cursos.

Un aspecto llamativo relacionado con la identificación de los stakeholders surgió del análisis de la terminología y de los conceptos que utilizaron muchas empresas que, en su mayoría, resultaron ser similares. Esto demostró, por un lado, el grado de racionalización que existe en ellas sobre estas cuestiones y, por el otro, el resultado del permanente benchmarking que realizan estas empresas, en particular con todo lo que tenga relación con RSE, sostenibilidad y stakeholders. Al respecto la Gerente de Sustentabilidad y Asuntos Públicos de la empresa $\mathrm{n}^{\circ} 12$ expresaba lo siguiente:

Identificamos a nuestros grupos de interés a partir de ejercicios de mapeos periódicos y los conocemos y nos relacionamos con ellos a partir de diferentes canales de comuni- 
cación unidireccionales y bidireccionales, los cuales nos permiten estar informados sobre sus inquietudes y necesidades, y escuchar sus sugerencias y expectativas; de manera que se puedan gestionar posibles riesgos y construir entre todos un negocio sustentable (Entrevista realizada el 22 de mayo de 2012).

Esta categoría, que emerge del análisis de los datos, se relaciona con los dos primeros objetivos de esta investigación. Se destaca, en consecuencia, el hecho de que prevalece en las empresas objeto del estudio la implantación de mecanismos de diferenciación y la escucha de las voces de los stakeholders como resultado de recomendaciones de organismos que desarrollan normas relacionadas con la RSE y la vinculación empresa-stakeholders. Si se asocia esta realidad a la notable dinámica manifestada por las empresas, en la participación de eventos o reuniones en los que varias de estas comparten sus experiencias y conocimientos sobre estos temas, puede inferirse que las compañías que adoptan estos mecanismos estandarizados sustentan su elección en el hecho de que encuentran en ellos herramientas de "buena administración", "aprobados" por organismos especializados, y utilizados mayoritariamente en las empresas.

\section{Participación de los stakeholders en los mecanismos organizacionales}

La vinculación entre las empresas y la sociedad transita por una evolución que, en cada estadio, responde a las características y circunstancias propias de cada época. Así, cuando en el inicio del desarrollo de las empresas se las consideraba como máquinas especializadas al servicio de sus propietarios, no resultaba extraño que la consciencia colectiva aceptara que esas organizaciones solo tuvieran como objetivo la maximización de la riqueza de quienes eran sus dueños. Al evolucionar desde una concepción mecanicista hacia una socio-sistémica, gradual y naturalmente se fueron incorporando a ese objetivo inicial intereses y deseos de otros partícipes tanto directos como indirectos. A pesar de esta evolución natural, el objetivo prioritario de la empresa sigue siendo la obtención de resultados económicos a través de la eficiencia en términos de ingresos y costos, para poder proporcionar a sus miembros los incentivos necesarios, que no solo son monetarios.

Hoy, las sociedades están frente al incipiente desarrollo de otro modelo de empresa, uno en el cual todos y cada uno de sus integrantes reconoce los efectos y consecuencias de 
sus acciones sobre los demás, resolviendo la oposición entre el interés propio y el ajeno, entre egoísmo y altruismo, actuando desde una posición que supera dichos dilemas. Este es un modelo de empresa más inclusivo, en el cual cada grupo de stakeholders merece consideración por lo que representa en sí mismo y no solo por su capacidad para ser un medio o instrumento de otro grupo. Al considerar a los stakeholders como seres humanos, complejos por naturaleza, se comienza a poner la ética y la responsabilidad en el centro de una nueva forma de concebir a las empresas. En ella los stakeholders tienen una participación más activa en los procesos organizacionales.

Los planteamientos del párrafo anterior son sostenidos por empresas que hacen pública su adhesión a los principios de RSE y de gestión de stakeholders. Sin embargo, en el relevamiento efectuado se encontraron muy pocas empresas que hubieran generado espacios de participación en los procesos organizacionales a sus principales stakeholders. Parecería que la actitud que prevalece es la de restringir la participación de los stakeholders únicamente a las instancias del diálogo.

De hecho, solo pocas empresas, entre las estudiadas, establecen con los stakeholders, de manera conjunta, las cuestiones relacionadas con la materialidad de los asuntos que serán tratados en la agenda compartida. Si bien la mayoría de ellas declara que luego de los diálogos iniciales comunican sus decisiones a los stakeholders que son afectados por ellas, pareciera que todavía se encuentra lejana la posibilidad de que los principales stakeholders tengan influencia en el armado de la agenda de los temas importantes de las compañías.

Esta realidad muestra que los directivos, que son a final de cuentas quienes deciden sobre las cuestiones que la empresa atenderá, del conjunto de temas que los stakeholders puede haberle hecho llegar, ejercen su discrecionalidad seleccionando libremente los asuntos en los que la compañía dedicará sus esfuerzos de gestión stakeholder. De esta manera, muchos directivos generan la ilusión de estar realizando un gobierno pluralista, cuando en realidad "maquillan" un proceso que sigue ajustándose a la lógica empresarial de gestión del "yo ordeno, mando y controlo".

Esta realidad no valida la proposición básica de esta investigación, asociada a los objetivos establecidos para ella, puesto que, a pesar de que la mayoría de las empresas tiene 
instrumentos administrativos institucionalizados para diferenciar y escuchar los intereses, deseos y objetivos de sus stakeholders, en lo sustancial sigue prevaleciendo la discrecionalidad por parte de los directivos en la elección de aquellos asuntos que la empresa atiende.

\section{El pensar, el decir y el hacer}

El fenómeno de la RSE y de la gestión de stakeholders se instaló en las grandes empresas del territorio argentino en los últimos años, de manera que su desarrollo puede ser calificado como "embrionario". Muchas grandes empresas sintieron la necesidad de incorporar de manera rápida aspectos de gestión relacionados con el enfoque stakeholder y con una actuación socialmente responsable. La prontitud por mostrar aspectos de gestión empresarial responsable se observa en ciertas conductas organizacionales, tales como aquellas en las que las empresas dicen lo que hacen con las demandas de los stakeholders, lo cual contrasta con lo que en realidad hacen con ellas, más específicamente, cuando se argumenta que estos deseos e intereses son considerados para ser incorporados en la visión, misión, estrategia y políticas de la empresa.

Así, la empresa $n^{\circ} 1$, que manifiesta que los intereses, deseos, deman- das, peticiones y expectativas de sus stakeholders son incorporados en la visión, misión, estrategia y políticas de la compañía, presenta en su página web el siguiente enunciado de misión y visión:

\section{Misión:}

Somos una empresa automotriz moderna y flexible, fabricante y vendedora de vehículos, transmisiones, componentes y repuestos, que brinda la mejor oferta de productos y servicios integrales para cada segmento y mercado en los que elegimos participar, satisfaciendo las necesidades de nuestros clientes basándonos en nuestros valores y los principios de procesos robustos, productos maduros y excelencia en atención.

\section{Visión:}

Ser una compañía automotriz integral, integrada al Grupo [...] rentable y sustentable ante escenarios cambiantes, basada en nuestros valores.

Ser el representante del Grupo [...] en el mercado de habla hispana de Sudamérica, produciendo $\mathrm{y}$ vendiendo productos y servicios tecnológicamente avanzados y confiables, excediendo las expectativas del cliente, liderando el mercado nacional e incrementando las exportaciones.

Ser el mejor equipo humano en el sector automotriz, asegurando el crecimiento y desarrollo de la gente. 
Como se puede apreciar, ni en la misión ni en la visión aparece reflejado el interés de esta empresa por incorporar en esos elementos los objetivos y deseos de los stakeholders. Este divorcio entre el pensar en hacer algo, decirlo y hacerlo concretamente es la constante que se encontró en la mayoría de las empresas. Este es un ejemplo de lo indicado antes sobre el estado incipiente de estos temas en las empresas argentinas. Otro ejemplo lo ofrece la empresa $\mathrm{n}^{\circ} 09$, cuya misión y visión se expresa de la siguiente manera:

\section{Misión:}

Prestar servicios financieros integrales y de excelencia con base en la calidad, colaboradores altamente capacitados y orientación hacia la satisfacción de nuestros clientes, buscando rentabilidad y contribuyendo al desarrollo sustentable del país.

\section{Visión:}

Ser el mejor banco privado de la Argentina.

Esta empresa manifiesta actuar con ciertos valores tales como: "cercanía, para construir relaciones de confianza a largo plazo; compromiso, para cumplir con nuestros objetivos; innovación, ofreciendo soluciones prácticas y adecuadas; entusiasmo, actuando en equipos de alto rendimiento". Además, declara volcar en su gestión las demandas de todos sus stakeholders. Pero, tanto de la lectura de su misión y visión como del conocimiento de sus estrategias, resulta evidente su falta de preocupación explícita por satisfacer los objetivos de otros stakeholders que no sean sus accionistas, para lo cual, tal como lo declara su misión, van a fijar su atención en sus colaboradores y clientes, valiéndose de ellos de una forma estratégico-instrumental, utilizándolos como medios para lograr su fin último: la maximización de ganancias para sus accionistas.

Sin embargo, en una de las empresas estudiadas se encontró una gestión en la cual había una congruencia total entre el pensar, el decir y el hacer. La empresa $n^{\circ} 15$ se presenta así en sus comunicaciones:

\section{Misión:}

Ser una empresa líder mundial en la cadena de agronegocios en términos de productos, servicios y conocimientos, orientada hacia el desarrollo y bienestar de las personas y su entorno. Queremos agregar valor a los negocios, transformar los sueños en valor sustentable y construir juntos el futuro.

\section{Visión:}

Crear el mejor ambiente para el desarrollo de las personas y su entorno, para ello proveemos materias primas, servicios e información a 
la red de agronegocios. Aspiramos a ser el mejor aliado en la cadena, por nuestra capacidad para interpretar y aceptar nuevos paradigmas y convertir las ideas en productos y servicios innovadores.

\section{Enunciación de valores o conceptos asociados:}

Pasión por hacer.

Inteligencia para darnos cuenta.

Generosidad para compartir.

En esta empresa se pudo constatar una preocupación sincera en relación con la gestión de stakeholders, siendo que muchos de ellos de manera directa, o a través de representantes, actúan en comités de asesoramiento al máximo órgano de dirección de la empresa. Esta fue la única empresa donde pudo verificarse la creación de verdaderos espacios de participación de los stakeholders en procesos organizacionales.

Del análisis de los datos revelados surge que, a pesar de la implantación de instrumentos estandarizados para detectar los intereses y deseos de los stakeholders, muchas empresas no los vuelcan en sus instrumentos de gestión ni en sus enunciados de objetivos. Si se acepta la prueba de efectividad de los mecanismos utilizados para diferenciar y escuchar a los stakeholders con la escasa presencia de sus intereses y objetivos expresados en instrumentos de las empresas, puede concluirse la poca efectividad de esos mecanismos para tales fines.

\section{E. Consideración estratégica de la actuación socialmente responsable}

Los intentos por integrar los conceptos de RSE con la estrategia corporativa incluyen el modelo de gestión estratégica de los grupos de interés y la inclusión de las demandas sociales como cuestiones estratégicas e instrumentales. En el primer caso, se examina la atención que presta la empresa a sus diversos stakeholders con el fin de lograr consecuencias positivas en el desempeño financiero de la empresa. El enfoque estratégico-instrumental responde, en cierta forma, a las actuales necesidades de legitimación de las empresas ante la sociedad, de manera que se presenta como "políticamente más correcto" que, por ejemplo, el enfoque accionarial puro, siendo que, al igual que este, aquel prioriza la creación de valor económico a largo plazo para los accionistas, pero teniendo en cuenta las restricciones impuestas por la necesidad práctica de satisfacer en una medida razonable los intereses, deseos y expectativas de los stakeholders no accionariales. Este enfoque se incorpora naturalmente a las ideas desarrolladas sobre estrategia 
empresarial que, con el objetivo de maximizar en el largo plazo la riqueza de los accionistas, nunca dejó de considerar que las empresas debían estar atentas a los objetivos sociales, pero considerando que estos tenían una influencia secundaria sobre los comportamientos directivos.

Siguiendo a Burke y Logsdon (1996) la RSE - política, programa o proceso- es estratégica cuando aporta beneficios a la empresa vinculados con el negocio, especialmente cuando apoya actividades consideradas como centrales y que contribuyen a la efectividad de la empresa en lograr su misión. Una reorientación estratégica de la empresa, en relación con su filosofía de responsabilidad social, apoya sus intereses financieros así como los de sus stakeholders. La reorientación de la RSE con una perspectiva más estratégica es la clave para que se realicen más actividades de responsabilidad social mientras se atienden los intereses de los stakeholders de una forma más integral. La idea implícita es que para que la RSE sea estratégica debe estar relacionada con la misión de la empresa y, en consecuencia, con su plan estratégico.

Desde esta perspectiva, en el estudio se encontró que la mayoría de las empresas consideraban su actuación socialmente responsable y la gestión de sus grupos de interés con un enfoque netamente estratégico. Sin embargo, los beneficios concretos buscados variaban entre ellas, entre otros, se encuentran gestión de riesgos, reducción de costos, incremento de la legitimidad, mejora de la imagen y la reputación de la empresa, y obtención de ventajas competitivas a través de la diferenciación con sus competidores.

En este sentido, y sin que fuera un objetivo establecido por la investigación, pudo detectarse que en todas las empresas estudiadas existía una necesidad concreta de darle visibilidad a sus actividades sociales y su gestión de stakeholders. La visibilidad se vincula con el grado en que las iniciativas sociales pueden ser observadas por los stakeholders de la empresa: los proyectos visibles de RSE parecen tener un efecto positivo en la reputación de la compañía. Según Roberts y Dowling (2002, p. 1078), algunas investigaciones han demostrado que una buena reputación empresarial tiene un importante potencial de creación de valor siendo además muy difícil de imitar por los competidores. La visibilidad de los programas de RSE permitiría que la empresa logre diferenciarse de sus competidores y, de esta forma, pueda crear valor bien sea a través del aumento de la cuota de mercado que pudiera 
obtener, o por medio de la obtención de una prima en el precio de sus productos.

La consideración de la importancia estratégica de la gestión de stakeholders y la actuación socialmente responsable se verificó en la investigación en el hecho de que solo en dos empresas el máximo cuerpo directivo no interviene en las estrategias, políticas y acciones orientadas en ese aspecto. Si bien en todas las demás empresas existen sectores o departamentos específicos relacionados con los temas de responsabilidad social y stakeholders, las líneas definitivas de las actuaciones se definen en lo más alto de la jerarquía organizacional. Un ejemplo de ello es lo expresado por el gerente de responsabilidad social corporativa de la Empresa n ${ }^{\circ}$ 09, a saber:

La Gerencia de Responsabilidad Social Corporativa instrumenta las políticas definidas por el Directorio y el Gerente General. Está compuesta por un equipo de siete personas y estructura su trabajo bajo tres dimensiones: capacitación, comunicación y coordinación. Dentro del organigrama, la misma es parte de la Gerencia de Área de Desarrollo Organizacional y Recursos Humanos. Sin embargo, tiene relación directa con el Directorio y la Gerencia General (Entrevista realizada el 19 de junio de 2012).

\section{F. Voluntariedad de las acciones}

Tal como se planteó anteriormente, las empresas que fueron objeto de estudio son aquellas que declaran públicamente tener un comportamiento socialmente responsable, a través de acciones sostenibles y con preocupación por sus stakeholders. Cabe destacar que en la República Argentina no existe legislación positiva que obligue a las empresas a realizar acciones o tener programas de responsabilidad social, de manera que las empresas que desarrollan actividades en ese sentido las realizan de manera voluntaria.

A pesar de no estar contemplado en ningún objetivo de esta investigación, el estudio determinó que la totalidad de las empresas realizan acciones de responsabilidad social y manifiestan considerar a sus stakeholders de manera voluntaria, sin recibir algún tipo de presión ni del sector industrial ni de la sociedad en general.

Esta característica, que presentan las empresas estudiadas, coincide con una de las dimensiones que Burke y Logsdon (1996) identificaron de los programas estratégicos de responsabilidad social de las empresas que sirven tanto a los intereses económicos de la empresa como a los de sus stakeholders, es decir, a la creación 
de valor. Ellos identificaron cinco dimensiones: la centralidad, la visibilidad, la voluntariedad, la especificidad y la proactividad.

La voluntariedad, según Burke y Logsdon(1996), requiere que la empresa desarrolle actividades sociales libremente, en lugar de responder a restricciones legales, incentivos fiscales o prácticas de la industria. En la literatura de RSE la voluntariedad se asocia con la elección. La elección se define en términos de la capacidad de superar las restricciones legales y fiscales (McWilliams \& Siegel, 2001). Aquellas empresas que optan por involucrarse en conductas voluntarias de RSE pueden aprovechar la oportunidad de construir recursos y capacidades específicas de la empresa, necesarias para la creación de valor. Además, la comunidad valora más las acciones voluntarias que las no voluntarias.

De las entrevistas realizadas a diferentes stakeholders de las empresas estudiadas se pudo verificar que no existe un total conocimiento de los actores respecto a que las acciones socialmente responsables de las empresas se realizan de manera voluntaria. Una vez conocido ese hecho, la valoración hacia ellas se tornó más positiva.

\section{CONCLUSIONES}

En los últimos años, dentro de la sociedad argentina se fortaleció un discurso social más inclusivo que le pide a las empresas que asuman un mayor compromiso con el crecimiento y con el desarrollo social. Sin embargo, la forma en la que las empresas toman estas nuevas obligaciones aún no se encuentra consolidada. Dirigir a las empresas, ampliando sus límites de gestión organizacional hacia la consideración de los grupos de interés es, por lo tanto, un fenómeno reciente y aún no cabalmente aceptado ni entendido por gran parte de los empresarios. Por ello, esta investigación propuso indagar sobre la forma y métodos que las empresas utilizan para relacionar las prácticas de responsabilidad social empresarial con la gestión de los stakeholders y el grado de institucionalización formal de esta gestión en las empresas a través de sus construcciones administrativas (identificándolas y evaluando su efectividad).

En el desarrollo de la investigación se pudo realizar el análisis de elementos correspondientes a los objetivos planteados y a la proposición de investigación asociada a ellos. En este sentido, y con respecto a la diferenciación de los stakeholders, 
resultó llamativa la poca refinación utilizada, ya que la identificación es solo nominal y no se utilizan indicadores cualitativos que permitan enriquecer esas clasificaciones.

En cuanto a los procedimientos organizacionales relacionados con la identificación y escucha de los stakeholders, los mecanismos utilizados mayormente provienen de recomendaciones de organismos internacionales que elaboran normas al respecto. Su implementación se sustenta en la utilización de estos mecanismos por la mayoría de las empresas que tienen inquietud por estos temas, de modo que las empresas, al utilizarlos, sienten que están aplicando herramientas de "buena administración". Tal vez el aspecto más destacable es que las empresas implementaron estos procedimientos respondiendo a situaciones concretas de reclamación de stakeholders, lo que evidencia una gestión de tipo reactivo más que proactivo.

Salvo en una empresa, la participación de los diferentes stakehold ers está limitada a ser actores de rondas de diálogo, en las cuales, si bien se los escucha, no se los considera luego en ninguna etapa de gestión que se haya generado a partir de esos diálogos. Pareciera que las instancias de participación otorga- das a los stakeholders se agotan en los diálogos.

Con esta realidad se determina que la proposición de investigación asociada a los objetivos de este trabajo no se verifica, ya que siguen siendo los directivos los que discrecionalmente establecen los temas y asuntos relacionados con los stakeholders que la empresa atiende.

En la investigación se identificó la existencia de divergencias muy claras entre lo que las empresas piensan que hacen, lo que dicen y lo que hacen realmente. Este divorcio se expresa de manera clara al estudiar sus manifestaciones públicas. Resulta probable que ese comportamiento esté relacionado con el estado embrionario de estas temáticas en Argentina.

A pesar de no ser un objetivo de la investigación, en ella se encontró que el modelo prevaleciente en las empresas es el de la consideración estratégica de la RSE. Por esta razón, su implementación presupone por parte de las compañías que en algún momento futuro las acciones realizadas traerán algún tipo de beneficio traducido en menores costos, mejora de imagen y reputación, ventaja competitiva, entre otros.

Finalmente, las acciones establecidas son impulsadas voluntariamente 
por las empresas, ya que no existe en el país legislación positiva sobre estas cuestiones.

\section{REFERENCIAS}

Argenti, J. (1970). Planificación de la empresa. Barcelona: OikosTau.

Burke, L., \& Logsdon, J. M. (1996). How corporate social responsibility pays off. Long Range Planning, 29(4), 495-502.

Elkington, J. (1994). Towards the sustainable corporation: Winwin-win business strategies for sustainable development. $\mathrm{Ca}$ lifornia Management Review, 36(2), 90-100.

Fassin, Y. (2012). Stakeholder management, reciprocity and stakeholder responsibility. Journal of Business Ethics, 109(1), 83-96.

Fassio, A., Pascual, L., \& Suárez, F. M. (2004). Introducción a la metodología de la investigación. Aplicada al saber administrativo y al análisis organizacional. Buenos Aires: Macchi.

Freeman, R. E. (1984). Strategic management. A stakeholder approach. Boston: Pitman Publishing.

Hayek, F. A. (1960/1985). The corporation in a democratic society: In whose interest ought it and will it be run? En Anshen, M., \& Bach G. L. (Eds.) (1985).
Management and corporations. Nueva York: McGraw-Hill.

International Organization for Standardization (2010). Norma ISO 26000:2010. Buenos Aires: IRAM.

Jensen, M. C. (2002). Value maximization, stakeholder theory, and the corporate objective function. Business Ethics Quarterly, 12(2), 235-256.

McWilliams, A., \& Siegel, D. (2001). Corporate social responsibility: A theory of the firm perspective. Academy of Management Review, 26(1), 117-127.

Mitchell, R., Agle, B., \& Wood, D. (1997). Toward a theory of stakeholder identification and salience: Defining the principle of who and what really counts. Academy of Management Review, 22(4), 853-886.

Pfeffer, J., \& Salancik, G. R. (1978/2003). The external control of organizations: A resource dependence perspective. California: Standord University Press. Recuperado de http://books.google. com.ar/books?id=iZv79yE-A C \& p g = P R $3 \& \mathrm{~h} 1=\mathrm{es} \&$ source $=$ gbs_selected_pages\&ca $\mathrm{d}=3 \# \mathrm{v}=$ onepage $\& \mathrm{q} \& \mathrm{f}=$ false

Ricart, E. J., Rodríguez, M. A., Blasco, J. L., Elorriaga, J. F., \& Castilla, M. L. (2002). Código de gobierno para la empresa sostenible. Madrid: Fundación Entorno. 
Roberts, P. W., \& Dowling, G. R. (2002). Corporate reputation and sustained superior financial performance. Strategic Management Journal, 23(12), 1077-1093.

Rodríguez F., J. M. (2002). Teoría de los partícipes y ciudadanía empresarial: una perspectiva pluralista del gobierno de las compañías. Ekonomiaz, $50\left(2^{\circ}\right.$ cuatrimestre), 74-109.

Stiglitz, J. (2004). Business social responsibility. En BSR 2004 Annual Conference, Nueva York. Recuperado de http://www.bsr. org/BSRConferences/2004/keynotes/2004_jstiglitz.pdf

Sturzenegger, A., Flores Vidal, M., \& Sturzenegger, G. (2003). Hacia una cultura de la respon- sabilidad social empresaria en Argentina. Buenos Aires: Foro Ecuménico Social.

Taylor, S. J., \& Bogdan, R. (1987). Introducción a los métodos cualitativos de investigación. Barcelona: Paidós Ibérica.

Valles M., M. (2000). La grounded theory y el análisis cualitativo asistido por ordenador. En García Ferrando, M., Ibañez, J., \& Alvira, F. (Comps.) (2000). El análisis de la realidad social. Métodos y técnicas de investigación (pp. 212-219). Madrid: Alianza.

Volpentesta, J. R. (2011). Gestión de la responsabilidad social empresaria ( $2^{\mathrm{a}}$ ed.). Buenos Aires: Librería Editorial Osmar Buyatti. 


\section{ANEXO}

Tabla 1. Detalle de las características configurativas de las empresas estudiadas*

\begin{tabular}{|c|c|c|c|c|c|c|c|c|c|c|c|c|c|c|c|c|c|c|c|c|c|c|c|c|c|}
\hline Empresas & 1 & 2 & 3 & 4 & 5 & 6 & 7 & 8 & 9 & 10 & 11 & 12 & 13 & 14 & 15 & 16 & 17 & 18 & 19 & 20 & 21 & 22 & 23 & 24 & 25 \\
\hline \multicolumn{26}{|l|}{ Tipo de empresa } \\
\hline Productiva & $\mathrm{X}$ & & $\mathrm{X}$ & & & & & & & $\mathrm{x}$ & & $\mathrm{X}$ & $\mathrm{X}$ & $\mathrm{X}$ & $\mathrm{X}$ & & & & $\mathrm{X}$ & & & $\mathrm{X}$ & $\mathrm{X}$ & & $\mathrm{X}$ \\
\hline Comercial & & & & & & & & & & & & & & & & & $\mathrm{X}$ & & & & & & & & \\
\hline De servicios & & $\mathrm{X}$ & & $\mathrm{X}$ & $\mathrm{X}$ & $\mathrm{X}$ & $\mathrm{X}$ & $\mathrm{X}$ & & & $\mathrm{X}$ & & & & & & & $\mathrm{X}$ & & $\mathrm{X}$ & $\mathrm{x}$ & & & $\mathrm{X}$ & \\
\hline Otras & & & & & & & & & $\mathrm{X}$ & & & & & & & $\mathrm{x}$ & & & & & & & & & \\
\hline \multicolumn{26}{|l|}{$\begin{array}{l}\text { Origen del } \\
\text { capital }\end{array}$} \\
\hline Nacional & & $\mathrm{X}$ & & & & $\mathrm{X}$ & & & $\mathrm{X}$ & & & & $\mathrm{x}$ & & $\mathrm{x}$ & & & & & & $\mathrm{x}$ & & & & $\mathrm{X}$ \\
\hline Extranjero & $\mathrm{X}$ & & $\mathrm{x}$ & $\mathrm{X}$ & $\mathrm{X}$ & & $\mathrm{X}$ & & & $\mathrm{x}$ & $\mathrm{x}$ & $\mathrm{X}$ & & $\mathrm{x}$ & & $\mathrm{x}$ & $\mathrm{X}$ & $\mathrm{x}$ & $\mathrm{x}$ & $\mathrm{x}$ & & $\mathrm{X}$ & $\mathrm{x}$ & $\mathrm{x}$ & \\
\hline Mixto & & & & & & & & $\mathrm{X}$ & & & & & & & & & & & & & & & & & \\
\hline \multicolumn{26}{|l|}{$\begin{array}{l}\text { Actividad } \\
\text { mercado interno }\end{array}$} \\
\hline \multicolumn{26}{|l|}{ Hasta el $25 \%$} \\
\hline \multicolumn{26}{|l|}{ Del 26 al $50 \%$} \\
\hline Del 51 al $75 \%$ & & & $\mathrm{X}$ & & & & & & & & & & & $\mathrm{X}$ & & & & & $\mathrm{X}$ & $\mathrm{X}$ & & & & & \\
\hline Más del $75 \%$ & $\mathrm{X}$ & $\mathrm{X}$ & & $\mathrm{X}$ & $\mathrm{X}$ & & & & & $\mathrm{X}$ & & $\mathrm{X}$ & $\mathrm{X}$ & & $\mathrm{X}$ & & & $\mathrm{X}$ & & & $\mathrm{X}$ & $\mathrm{X}$ & $\mathrm{X}$ & $\mathrm{X}$ & $\mathrm{X}$ \\
\hline $\begin{array}{l}\text { Solo mercado } \\
\text { interno }\end{array}$ & & & & & & $\mathrm{X}$ & $\mathrm{X}$ & $\mathrm{X}$ & $\mathrm{X}$ & & $\mathrm{x}$ & & & & & $\mathrm{x}$ & $\mathrm{X}$ & & & & & & & & \\
\hline \multicolumn{26}{|l|}{$\begin{array}{l}\text { Cantidad de } \\
\text { empleados }\end{array}$} \\
\hline \multicolumn{26}{|l|}{ Hasta 40} \\
\hline \multicolumn{26}{|l|}{ De 41 a 100} \\
\hline De 101 a 300 & & & $\mathrm{X}$ & & & & & & & & & & & & & & & & & & & & & & \\
\hline De 301 a 500 & & & & & & $\mathrm{X}$ & & & & & & & & & $\mathrm{X}$ & & & & & & & & $\mathrm{X}$ & & \\
\hline
\end{tabular}

* En la investigación se garantizó la absoluta confidencialidad de los datos, los que solo son utilizados con fines académicos. Existió un compromiso de que en ningún documento o escrito que pudiera surgir de la presente investigación se mencionarían los nombres de las empresas y la información solo sería presentada y, eventualmente publicada, en cuadros o gráficos consolidados o resúmenes. 
Identificación del impacto de la gestión de los stakeholders

\begin{tabular}{c|c|c|c|c|c|c|c|c|c|c|c|c|c|c|c|c|c|c|c|c|c|c|c|c|c}
\hline Empresas & 1 & 2 & 3 & 4 & 5 & 6 & 7 & 8 & 9 & 10 & 11 & 12 & 13 & 14 & 15 & 16 & 17 & 18 & 19 & 20 & 21 & 22 & 23 & 24 & 25 \\
\hline De 501 a 750 & & & & & & & $\mathrm{X}$ & $\mathrm{X}$ & & & & & & & & & & & & & & & & & \\
\hline De 751 a 1.000 & & & & & & & & & & & & & & & & & & & & & & & & \\
\hline De 1.001 a 1.500 & & & & & & & & & & & & $\mathrm{X}$ & & $\mathrm{X}$ & & & & & & & & & & & \\
\hline De 1.501 a 2.000 & & & & & & & & & & & & & & & & & & & & $\mathrm{X}$ & $\mathrm{X}$ & $\mathrm{X}$ & & & \\
\hline De 2.001 a 2.500 & & & & & & & & & & $\mathrm{X}$ & & & & & & & & & & & & & & & \\
\hline Más de 2.500 & $\mathrm{X}$ & $\mathrm{X}$ & & $\mathrm{X}$ & $\mathrm{X}$ & & & & $\mathrm{X}$ & & $\mathrm{X}$ & & $\mathrm{X}$ & & & $\mathrm{X}$ & $\mathrm{X}$ & $\mathrm{X}$ & $\mathrm{X}$ & & & & & $\mathrm{X}$ & $\mathrm{X}$ \\
\hline
\end{tabular}

Fuente: Elaboración propia. 